\title{
Segregation of DFNB49 with congenital sensorineural bilateral profound hearing
} loss

\author{
Nazir Ahmad ${ }^{1,2 *}$,Muhammad Qasim ${ }^{3}$, Zubair M. Ahmed ${ }^{1,4}$ and \\ Sheikh Riazuddin ${ }^{1}$ \\ 1- National Centre of Excellence in Molecular Biology, University of the Punjab Lahore Pakistan \\ 2- Department of Biology, Government Post Graduate College Civil Lines Multan Pakistan \\ 3- Department of Bioinformatics and Biotechnology, Government College University Faisalabad Pakistan. \\ 4- Department of Otorhinolaryngology Head \& Neck Surgery School of Medicine University of Maryland, USA \\ Corresponding Author: nazirahmad1976@yahoo.com
}

Citation

Nazir Ahmad, Muhammad Qasim, Zubair M. Ahmed and Sheikh Riazuddin. Segregation of DFNB49 with congenital sensorineural bilateral profound hearing loss. Pure and Applied Biology Vol. 4, Issue 2, 2015, pp 150-153.

Received: $11 / 04 / 2015$

Revised: $10 / 05 / 2015$

Accepted: $27 / 05 / 2015$

\section{Abstract}

Deafness is a complete or partial loss of hearing. In Pakistan, the prevalence of this order is high as compared to the other countries because of frequent consanguineous marriages in Pakistan. During present study, DFNB49 was mapped in two unrelated consanguineous families having multiple affected individuals. The maximum LOD score was found 4.71 and 2.74 for PKDF1069 \& PKDF751 respectively. This study reveals genetic heterogeneity of this clinically heterogeneous disorder.

Key Words: Deafness; DFNB49; Linkage Analysis.

Introduction

Deafness is one of the most common neurosensory, genetically and clinically heterogeneous disorders in the world, caused by hereditary as well as environmental factors. The occurrence of congenital severe to profound hearing loss is estimated to be 1 in 1000 newborns [1]. The segregation of genetic deafness may follow an autosomal dominant, autosomal recessive, $\mathrm{X}$-linked, $\mathrm{Y}$ linked or mitochondrial mode of inheritance. These monogenic forms of deafness may be non-syndromic (no other clinical phenotype in addition to hearing loss) or syndromic (characterized by loss of hearing in combination with other abnormalities like RP, Goiter, pigmentation abnormalities etc.) [2]. The syndromic forms account for $30 \%$ of hereditary hearing loss and include more than 400 reported deafness syndromes [3]. In non-syndromic genetic deafness of prelingual onset, autosomal recessive inheritance predominates (75-80\%), followed by autosomal dominant (12-24\%), X-linked $(1-3 \%)$ and mitochondrial $(<1 \%)$ $[4,5]$. Homozygosity results in the development of disease phenotype in autosomal recessive cases. Homozygosity is the function of consanguinity. The results of a demographic survey showed that $60 \%$ of the marriages in Pakistan are among blood relatives. Due to high breeding coefficient of Pakistani population, the incidence of deafness is high in Pakistan as compared to other countries of the world. This high consanguinity makes Pakistani population 
the best resource for genetic analysis of hereditary deafness [6].

The human inner ear contains a variety of cells that are essential for sound transduction [7]. Identification of genes segregating with deafness is difficult because of tremendous genetic and clinical heterogeneity of the disorder, small size of affected families and the uncontrolled mating which seriously hinder the chromosomal mapping and identification of the causative genes [8].

These problems can be overcome by linkage analysis on inbred families having at least three or more affected individuals that are likely to be genetically homogeneous. Linkage analysis is a powerful tool not only for mapping new genomic regions, but also for the positional cloning of previously mapped loci $[9,10]$. Genetic screening for reported loci not only refines the proximal and distal boundaries of reported regions but also paves the way to detect the pathogenic alleles in families whose disease phenotype is linked to the loci with already reported gene. During linkage studies, deafness phenotype segregating in two families showed linkage to the already reported recessive deafness locus DFNB49 (PKDF1069 and PKDF751).

This study will help to offer genetic counseling to the families to reduce the incidence of hereditary deafness in our population thereby decreasing socioeconomic burden.

\section{Materials and Methods}

The study was duly approved from Institutional Review Board. Families suffering from non-syndromic sensorineural hearing loss (NSSHL) were identified by a vast search from the schools and centers for special education situated in different cities of Punjab. The families with at least 3 or more affected individuals were selected for inclusion in study. These families were personally visited at their homes and their complete disease history and pedigrees were recorded. With the informed consent of participants, blood specimens of normal and affected individuals were collected. These samples were transported to genetic diseases laboratory of National Centre of Excellence in Molecular Biology by following standard protocols.

A total of 10 families with three or more deafness affected individuals were enrolled in this study. The samples of these families were subjected to DNA extraction using a standard phenol extraction protocol and used in the linkage analyses. At least three to four fully informative fluorescently labeled markers were used for every locus. These markers were amplified through PCR and were subjected to genotyping analysis. Genotyping data was analyzed and haplotypes were constructed to find the pattern of inheritance among the affected and normal individuals of each family under study. If the deafness phenotype in a family showed potential linkage to any of the known locus, more closely spaced STR markers were genotyped. Two-point LOD scores $(Z \max )$ were calculated for each family using MLINK software.

\section{Results}

\section{Linkage to $D F N B 49$}

\section{PKDF1069}

This pedigree was enrolled from "Jampur" and belongs to "Dhandra" caste. It contained five affected individuals in three loops. These affected individuals showed congenital, bilateral sensorineural hearing loss (Fig.1). Clinical analysis of PKDF1069 revealed no other extra auditory phenotype co-segregating with hearing loss. Deafness Phenotype segregating in PKDF1069 was linked to markers D5S2036 $(74.68 \mathrm{Cm})$, GATA-141B10 (75.89cM), D5S637 $(75.90 \mathrm{cM})$ and $D 5 S 1982(78.3 \mathrm{cM})$ that encompass the DFNB49 locus. Maximum two point LOD score of 4.71 and 4.69 were obtained for D5S2036 and D5S637 respectively .Parents of 3rd generation (III:1 \& III:4, III:2 \& III:3, III:6 \& III:7) and individuals IV:6,11 and IV:12 are carrier as they carry a disease allele for every marker but have no disease phenotype. 


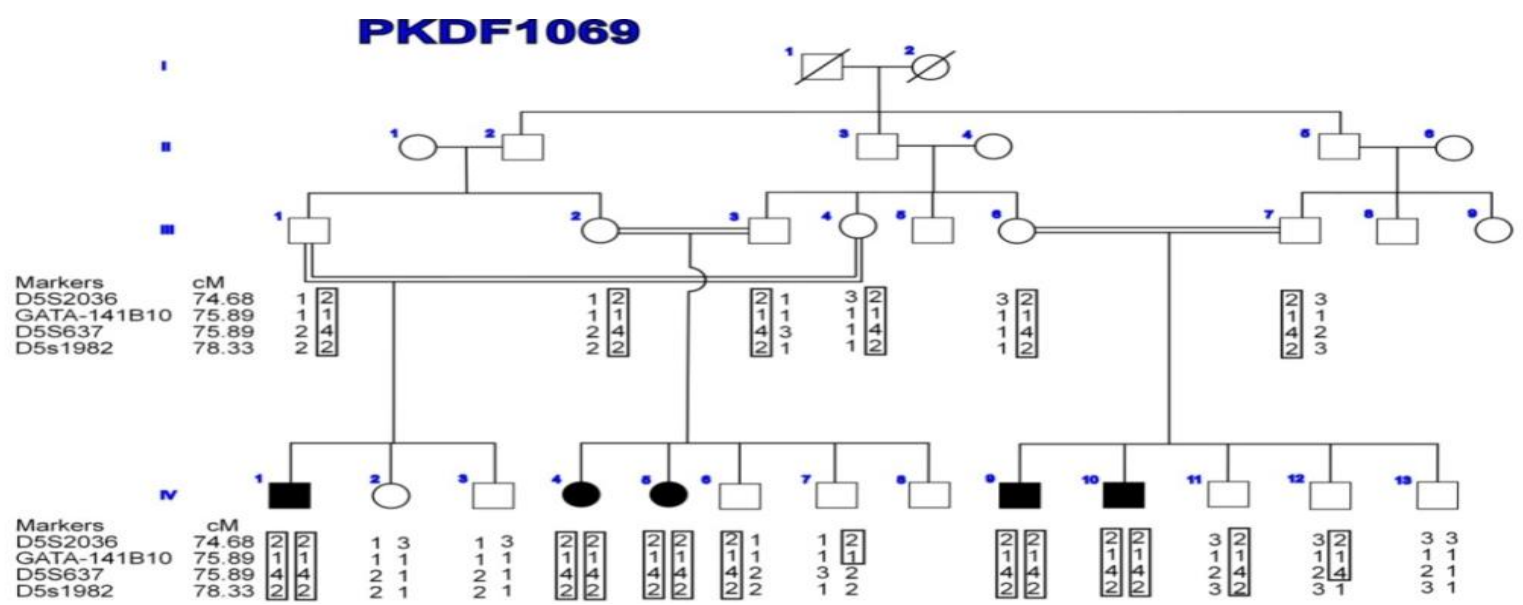

Fig. 1 DFNB49 linked haplotype in family PKDF1069 at chromosome 5. Black symbols represent disease phenotype. The genetic linkage markers and their relative positions in centimorgans (cM) according to the Marshfield maps are shown on the left side of the pedigree.

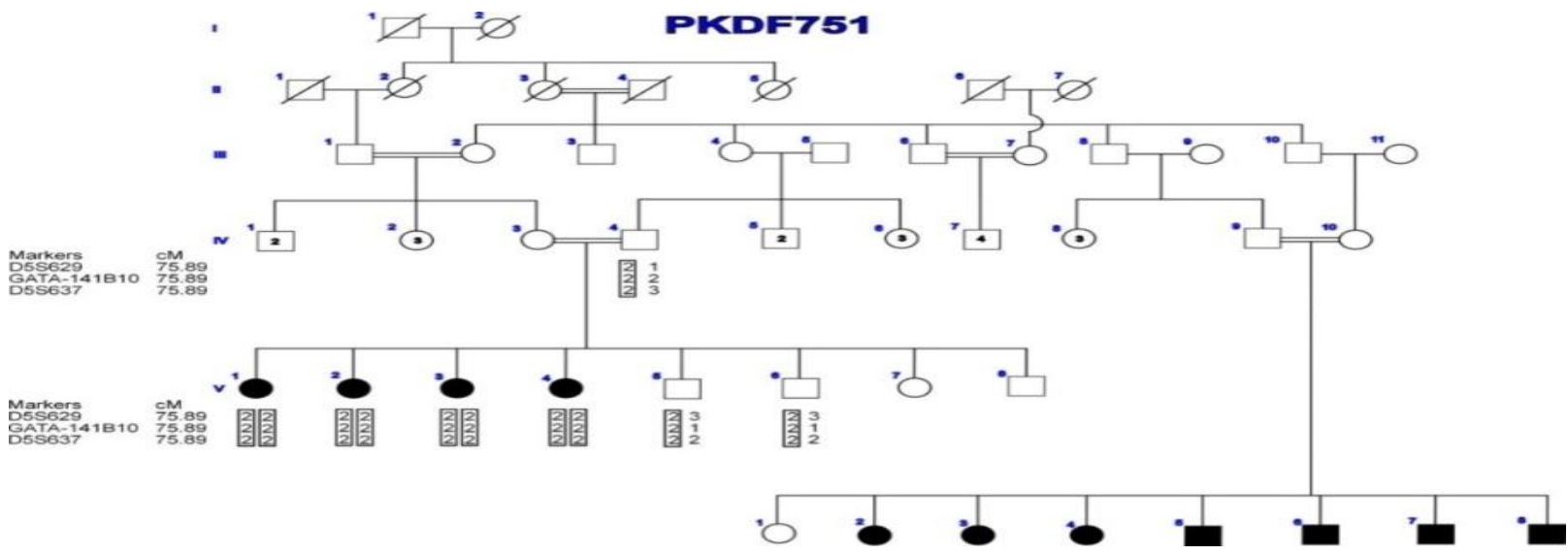

Fig-2. DFNB49 linked haplotype in family PKDF751 at chromosome 5. Black symbols represent individuals who are deaf. The genetic linkage markers and their relative positions in centimorgans (cM) according to the Marshfield maps are shown on the left side of the pedigree.

On the other hand the individuals IV: $2,3, \&$ IV:13 have neither disease phenotype nor any disease allele showing that they are not carrier.

\section{PKDF751}

This family belongs to caste "Arian" and was collected from "Gujranwala" district of Punjab. It has eleven live deaf individuals in two loops. Affected individuals had nonsyndromic deafness as no features of night blindness, goiter, pigmentation abnormalities and vestibular dysfunction were observed. The inheritance of deafness in the family is consistent with an autosomal recessive mode of inheritance (Fig.2).
Linkage analysis and haplotype construction of microsatellite markers D5S629, GATA$141 B 10$ and D5S637 showed that all the affected individuals were homozygous for these markers while the normal individuals were heterozygous. As these markers encompass the recessive deafness locus DFNB49 at chromosome 5, the family was considered linked. Maximum LOD score (Zmax) of 2.74 was obtained for the markers D5S629 (75.89 cM) and GATA-141B10 $(95.89 \mathrm{cM})$ at recombination fraction $\theta=0$. All the normal individuals of this family whose DNA samples were available were found carrier (III: 3, IV:1 and IV:6). 


\section{Discussion}

The linkage studies of hereditary hearing loss offer a unique canvas to draw two types of illustrations all together-First, identification of the causative genes and the underlying pathogenic process in each form of deafness and - Second, elucidation of the molecular and cellular mechanisms of hearing.

Haplotypes of two consanguineous pedigrees, PKDF1069 and PKDF751 were found linked to DFNB49/TRIC. DFNB49 was mapped in two Pakistani pedigrees on chromosome 5q12.3-q14.1 through genome wide scan [12]. Positional cloning of DFNB49 resulted in the identification of pathogenic variants of TRIC gene [13]. Segregation of deafness phenotype due to this gene mutation has not yet been reported in any other population except Pakistan.

\section{Conclusion}

Linkage analysis is an effective technique in mapping disease causing loci and to determine the carrier status of individuals. Genetic counseling can be provided to these families to reduce the incidence of disease in next generations.

\section{Acknowledgements}

We are thankful to teaching staff of schools and centers of special education and especially the deaf families for their voluntary participation in the study.

\section{References}

1. Atar O, \& Avraham KB (2005). Therapeutics of hearing loss: expectations vs reality. Drug Discov. Today 10, 1323 -1230. 2. Kalatzis V, \& Petit C (1998). The fundamental medical impacts of recent progress in research on hereditary hearing loss. Hum Mol Genet 7: 1589-1597.

3. Gorlin RJ, Toriello HV, Cohen MM (1995). Hereditary Hearing Loss and its Syndromes. Oxford University Press, New York.
4. Petersen MB, \& Willems PJ (2006). Non-syndromic, autosomal recessive deafness. Clin Genet: 69:371 -392.

5. Morton CC, \& Nance WE (2006). Newborn Hearing Screening - A Silent Revolution. N Engl J Med 354:2151-64.

6. Hussain R \&Bittles AH (1998). The prevalence and demographic characteristics of consanguineous marriages in Pakistan.

J BiosocSci 30:261-275.

7. Adato A, Michel V, Kikkawa Y, Reiners J, Alagramam, KN, Weil D, Onekawa H, Wolfrum U, El-Amraoui A, PetiC.(2005).Interactions in the network of usher syndrome type 1 proteins. Hum Mol Genet 14:347-356.

8. Van Camp G, Willems PJ \& Smith RJH (1997). Nonsyndromic hearing impairment unparalleled heterogeneity. Am J Hum Genet 60:758-764.

9. Friedman TB, Griffith AJ (2003). Human nonsyndromic sensorineural deafness. Annu Rev GenomicsHum Genet 4:341-402.

10. Woods CG, Cox J, Springell K, Hampshire DJ, Mohamed MD, McKibbin M , Stern R, Raymond F. L, Sandford R, Sharif S, Karbani G, Ahmed M, Bond J, Clayton D, Inglehearn CF (2006). Quantification of homozygosity in consanguineous individuals with autosomal recessive disease. Am J Hum Genet 78(5):889-96.

11. Ramzan K,Shaikh RS, Ahmad J, Khan SN, Riazuddin S, Ahmed ZM, Friedman TB, Wilcox ER, Riazuddin S (2005).A new locus for nonsyndromic deafness DFNB49 maps tochromosome 5q12.3-q14.1. Hum Genet 116:17-22.

12. Riazuddin S, Ahmed ZM, Fanning AS, Lagziel A, Kitajiri S, Ramzan K, Khan SN, Chattaraj P, Friedman PL, Anderson JM,Belyantseva IA, Forge A, RiazuddinS, Friedman TB(2006b). TRICELLULIN is a tight-junction protein necessary for hearing. Am J Hum Genet 79(6):1040-51. 